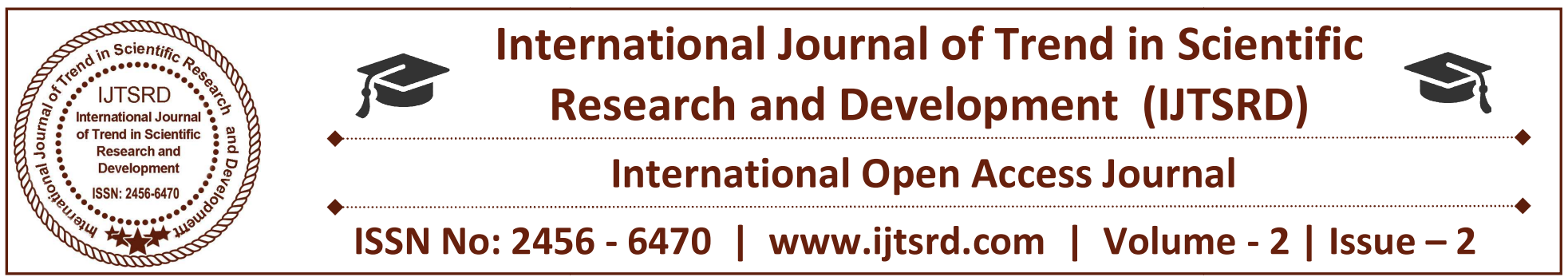

\title{
The Performance Analysis of PAPR Reduction using a SLM technique at different parameters $u$ and $M$ in OFDM-MIMO System
}

\author{
Er. Sukhjinder Singh \\ Astt. Prof. (HOD) EEE, MGC, \\ Mukerian, Punjab, India
}

\author{
Er. Parvinder Singh \\ Astt. Prof. EEE, MGC, \\ Mukerian, Punjab, India
}

\author{
Er. Jasraj Singh \\ Astt. Prof. EEE, MGC, \\ Mukerian, Punjab, India
}

\section{ABSTRACT}

The ecological functions of urban areas are degraded; the infrastructures of cities and the built-up regions have enormous impacts on ecosystems and ecology of the urban environment. In this paper, we focused on the connections of urban ecology and urban design, urbanisation impacts on urban ecosystems functions and ecological urbanism approach to degraded urban areas. The studies revealed the relationship of urban ecology and urban design and the ecosystem services they provide in the urban environment. In Orthogonal Frequency Division Multiplexing (OFDM) is an new method for fourth generation wireless communication. MIMO-OFDM has become a promising candidate for high performance $4 \mathrm{G}$ broadband wireless communications. However, one main disadvantage of MIMO-OFDM is the high peak-to-average power ratio (PAPR) of the transmitter's output signal on different antennas. In this paper, we present a new noble SLM PAPR reduction techniques such as selective mapping technique and Partial transmit sequence techniques and shows which of these PAPR reduction techniques are more effective to reduce PAPR in OFDM-MIMO.

Keywords: OFDM, SLM, PAPR, MIMO, ISI, BER, PAPR

\section{INTRODUCTION}

OFDM is one of the multicarrier modulation technique for 4th Generation (4G) wireless communication. This technique is a new technique for high-speed data transmission used in mobile communication, Digital terrestrial mobile communication, Digital Audio Broadcasting (DAB), Digital Video Broadcasting terrestrial (DVB-T). OFDM has many advantages such as robustness in frequency selective fading channels, High spectral efficiency immunity to inter-symbol interference and capability of handling very strong multipath fading. But OFDM is having major drawback of a high Peakto-Average Power ratio (PAPR).This causes clipping of the OFDM signal by the High power amplifier (HPA) and in the HPA output producing nonlinearity. This non-linearity distortion will result in-band distortion and out-of-band radiation. The in-band distortion causes system performance degradation and the out-of-band radiation causes adjacent channel interference (ACI) that affects systems working in neighbour band. Hence the OFDM signal may have In-band and Out-of-band distortion which degradation of Bit-error-rate (BER) performance. One solution is to use a linear power amplifier with large dynamic range. However, most promising solution to reduce PAPR by using through PAPR reduction techniques such as SLM and PTS.

\section{ORTHOGONAL FREQUENCY DIVISION MULTIPLEXING (OFDM):}

OFDM is a method of encoding digital data on multiple carrier frequencies. OFDM has developed into a popular scheme for wide band digital communication, whether wireless or over copper wires, used in applications such as digital television 
and audio broadcasting, DSL broadband internet access, wireless networks, and 4G mobile communications. OFDM is essentially identical to coded OFDM (COFDM) and discrete multi-tone modulation (DMT), and is a frequency-division multiplexing (FDM) scheme used as a digital multicarrier modulation method. A large number of closely spaced orthogonal sub-carrier signals are used to carry data. The data is divided into several parallel data streams or channels, one for each sub-carrier. Each sub-carrier is modulated with a conventional modulation scheme (such as quadrature amplitude modulation or phase-shift keying) at a low symbol rate, maintaining total data rates similar to conventional single-carrier modulation schemes in the same bandwidth. The primary advantage of OFDM over single-carrier schemes is its ability to cope with severe channel conditions (for example, attenuation of high frequencies in a long copper wire, narrowband interference and frequency-selective fading due to multipath) without complex equalization filters. Channel equalization is simplified because OFDM may be viewed as using many slowly modulated narrowband signals rather than one rapidly modulated wideband signal. The low symbol rate makes the use of a guard interval between symbols affordable, making it possible to eliminate intersymbol interference (ISI) and utilize echoes and timespreading (that shows up as ghosting on analogue TV) to achieve a diversity gain, i.e. a signal-to-noise ratio improvement. This mechanism also facilitates the design of single frequency networks (SFNs), where several adjacent transmitters send the same signal simultaneously at the same frequency, as the signals from multiple distant transmitters may be combined constructively, rather than interfering as would typically occur in a traditional single-carrier system.figl. shows an OFDM spectrum
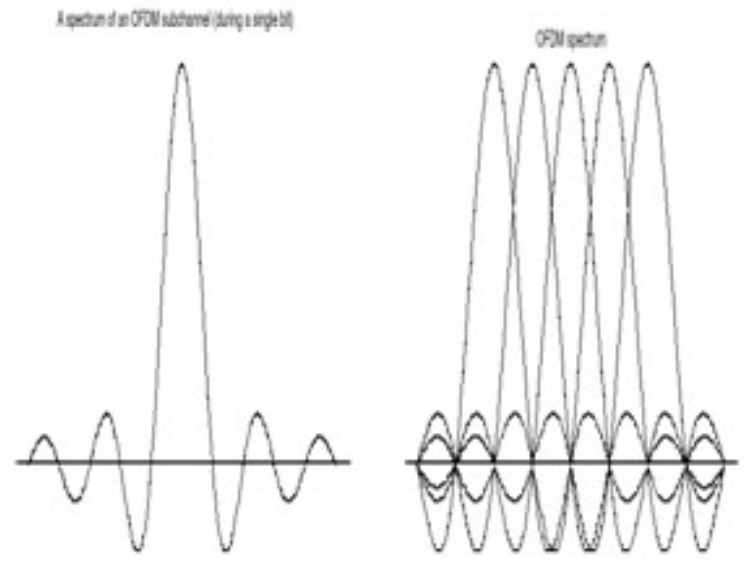

\section{Orthogonality}

In OFDM, the sub-carrier frequencies are chosen so that the sub-carriers are orthogonal to each other, meaning that cross-talk between the sub-channels is eliminated and inter-carrier guard bands are not required as shown in OFDM spectrum. This greatly simplifies the design of both the transmitter and the receiver; unlike conventional FDM, a separate filter for each sub-channel is not required.

\section{OFDM TRANSCEIVE STRUCTURE}

To generate OFDM successfully the relationship between all the carriers must be carefully controlled to maintain the orthogonality of the carriers. For this reason, OFDM is generated by firstly choosing the spectrum required based on the input data, and modulation scheme used. Each carrier to be produced is assigned same data to transmit. The required amplitude and phase of them are calculated based on the modulation scheme. The required spectrum is then converted back to its time domain signal bin, thus performing the IFFT using an Inverse Fourier Transform (IFT). In most applications, an Inverse Fast Fourier Transform (IFFT) is used.

The IFFT performs the transformation very efficiently and provides a simple way of ensuring the carrier signals produced are orthogonal. The Fast Fourier Transform (FFT) transforms acyclic time domain signal into its equivalent frequency spectrum. This is done by finding the equivalent waveform, generated by a sum of orthogonal sinusoidal components. The amplitude and phase of the sinusoidal components represent the frequency spectrum of the time domain signal. The IFFT performs the reverse process, transforming a spectrum (amplitude and phase of each component) into a time domain signal. An IFFT converts a number of complex data points, of length that is a power of 2 , into the time domain signal of the same number of points. Each data point in frequency spectrum used for an FFT or IFFT is called a bin. The orthogonal carrier required for the OFDM signal can be easily generated by setting the amplitude and phase of each frequency.

Fig. 1 OFDM SPECTRUM 


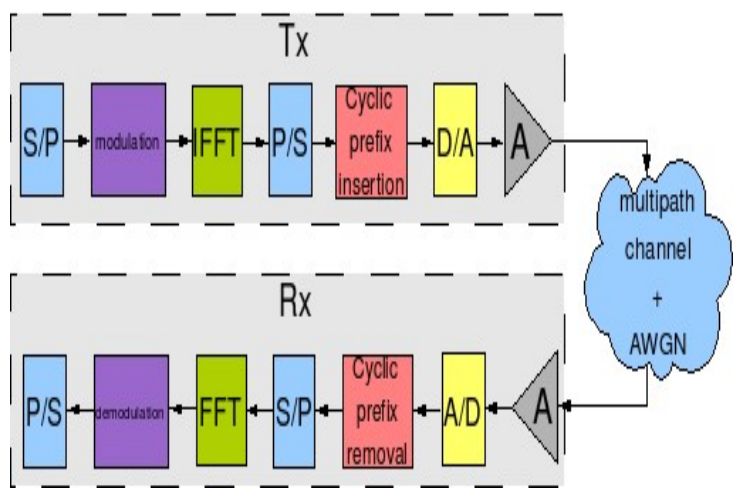

Fig.2. OFDM Transceiver Structure Fig.2 shows the configuration for a basic OFDM Transmitter and Receiver.

\section{PROBLEM OF PEAK-TO-AVERAGE POWER RATIO IN OFDM SYSTEM}

High Peak-to-Average Power Ratio (PAPR) has been recognized as one of the major practical problem in OFDM signal. High PAPR results from the nature of the modulation itself where multiple subcarriers $/$ sinusoids are added together to form the signal to be transmitted. When $\mathrm{N}$ sinusoids add, the peak magnitude would have a value of $\mathrm{N}$, Where the average might be quite low due to the destructive interference between the sinusoids. High PAPR signals are usually undesirable for it usually strains the analog circuitry. High PAPR signals would require a large range of dynamic linearity from the analog circuits which usually results in expensive devices and high power consumption with lower efficiency (for e.g. power amplifier has to operate with larger back-off to maintain linearity).

In OFDM system, some input sequences would result in higher PAPR than others. For example, an input sequence that requires all such carriers to transmit their maximum amplitudes would certainly result in a high output PAPR. Thus by limiting the possible input sequences to a smallest sub set, it should be possible to obtain output signals with a guaranteed low output PAPR.

\section{PAPR Definition}

Theoretically, large peaks in OFDM system can be expressed as Peak-to-Average Power Ratio, or referred to as PAPR, in some literatures, also written as PAR. It is usually defined as,

$$
P A P R=\frac{P_{\text {peak }}}{P_{\text {average }}}=10 \log _{10} \frac{\max \left[\left|x_{n}\right|^{2}\right]}{E\left[\left|x_{n}\right|^{2}\right]}
$$

Where Ppeak represents peak output power, Paverage means average output power. $E \cdot$ denotes the expected value, $x_{n}$ represents the transmitted OFDM signals which are obtained by taking IFFT operation on modulated input symbols. Mathematical, $x_{n}$ is expressed as:

$$
x_{n}=\frac{1}{\sqrt{N}} \sum_{k=0}^{N-1} X_{k} W_{N}^{n k}
$$

For an OFDM system with $N$ sub-carriers, the peak power of received signals is $N$ times the average power when phase values are the same. The PAPR of baseband signal will reach its theoretical maximum at $(d B)=10 \log N$. For example, for a 16 sub-carriers system, the maximum PAPR is $12 \mathrm{~dB}$. Nevertheless, this is only a theoretical hypothesis. In reality the probability of reaching this maximum is very low.

\section{DIFFERENT TECHNIQUES}

PAPR

REDUCTION

At 0 present, there are many PAPR reduction techniques of OFDM. Such as Clipping and Filtering, block coding, Sub block coding technique, Selective Mapping (SLM), Partial Transmit Sequences (PTS) Interleaving, Tone Reduction (TR), Tone Injection (TI). But out of these techniques SLM and PTS provides the efficient solution for PAPR reduction in OFDM -MIMO system. symbols. Therefore in the paper we study only one techniques and study the performances of SLM PAPR Reduction techniques.

\subsection{SELECTIVE MAPPING TECHNIQUE}

In selective mapping (SLM) technique the actual transmit signal lowest PAPR is selected from a set of sufficiently different signals which all represents the same information. . In the SLM technique, the transmitter generates a set of sufficiently different 
candidate data blocks, all representing the same most favourable for transmission information as the original data block, and selects the

Block diagram of SLM Technique is shown in Fig.2

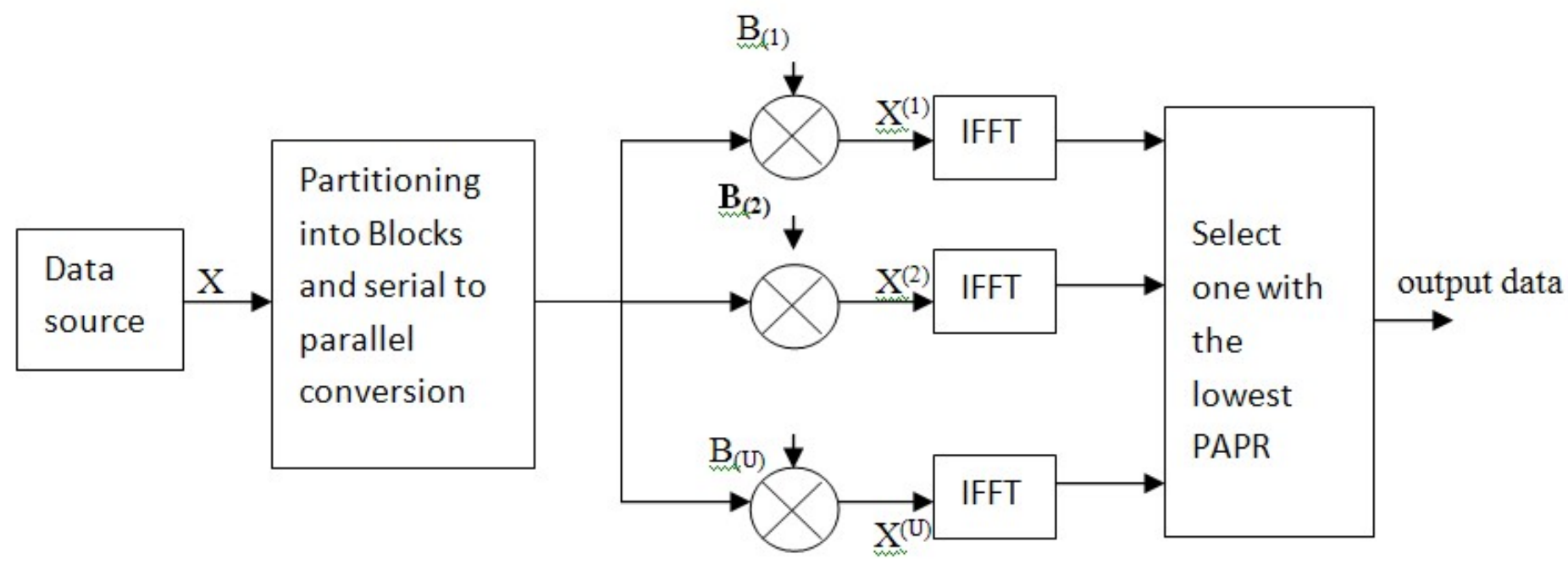

Fig. 2: Block Diagram of OFDM transmitter with the SLM Technique Let.s define data stream after serial to parallel here $\triangle \mathrm{f}=\frac{1}{N T}$, NT is the duration of an OFDM data
conversion as block. Output data of the lowest PAPR is selected to

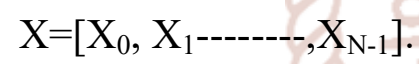

Each data block $\mathrm{X}=\left[\mathrm{X}_{0}, \mathrm{X}_{1}\right.$--------, $\left.\mathrm{X}_{\mathrm{N}-1}\right]$ is multiplied element by element with different phase sequences, each of length $N$. Initially each input $X_{n}{ }^{u}$ can be defined as equation

$$
\mathrm{X}_{\mathrm{n}}{ }^{\mathrm{u}}=\mathrm{X}_{\mathrm{n}} \cdot \mathrm{b}_{\mathrm{n}}{ }^{\mathrm{u}}
$$

$\mathrm{X}_{\mathrm{n}}{ }^{\mathrm{u}}$ and $\mathrm{b}^{\mathrm{u}}$ can be written as

$$
\begin{aligned}
& \mathrm{X}_{\mathrm{n}}^{\mathrm{u}}=\left[\mathrm{X}_{\mathrm{o}}^{\mathrm{u}}, \mathrm{X}_{1}{ }^{\mathrm{u}}, \ldots \ldots \ldots \ldots \ldots \ldots \mathrm{X}_{\mathrm{N}-1}{ }^{\mathrm{u}}\right] \\
& \mathrm{b}_{\mathrm{u}}=\left[\mathrm{b}_{\mathrm{u}, \mathrm{o}}, \mathrm{b}_{\mathrm{u}, 1}, \ldots \ldots \ldots \ldots \ldots \ldots \mathrm{b}_{\mathrm{u}, \mathrm{N}-1}\right]
\end{aligned}
$$

Where $\mathrm{n}=0,1,2------\mathrm{N}-1$, and $\mathrm{u}=0,1,2 \ldots \mathrm{U}$ to make the $U$ phase rotated OFDM data blocks. All $U$ phase rotated OFDM data blocks represented the same information as the unmodified OFDM data block, provided that the phase sequence is known. After applying the SLM technique, the complex envelope of the transmitted OFDM signal becomes

$\mathrm{X}(\mathrm{t})=\frac{1}{\sqrt{N}} \sum_{n=o}^{N-1} X_{n} e^{j 2 \Omega n \triangle f t} \quad 0<\mathrm{t}<\mathrm{NT}$ transmit. PAPR reduction effect will be better as the copy block number $U$ is increased. SLM method effectively reduce PAPR without any signal distortion. But it has higher system complexity and computational burden. This complexity can less by reducing the number of IFFT block. but this original SLM techniques don't give good result in PAPR reduction. Therefore we use new SLM technique with sub band permutation scheme in this paper which give better PAPR reduction results. In this paper we use Alamouti scheme (STBC) for the MIMO-OFDM system in the case of two transmitting antenna .we know that during the first time slot, two OFDM symbol transmitted from antennas 1 and 2 are given by, respectively

$$
\begin{aligned}
& \mathrm{X}_{1}=\left[X_{0}^{1}, X_{1}^{1}-------, X_{N-1}^{1}\right], \\
& \mathrm{X}_{2}=\left[X_{0}^{2}, X_{1}^{2} \text {------- }, X_{N-1}^{2}\right],
\end{aligned}
$$

During the next time slot, complex conjugate $-X_{2}$ is transmitted from antenna 1 , and $X_{1}$ is transmitted from antenna 2. For an MIMO-OFDM system with 2 transmit antennas and $N$ subcarriers . total subcarriers 
are divided into $M$ subbands of equal sizes, Then, we find the average PAPR of $X_{1}$ and $X_{2}$ and represented as

$\mathrm{X}_{\mathrm{j}}=\left[X_{1}^{j}, X_{2}^{j}------, X_{M}^{j}\right]$

Where $j$ denotes the index of transmit antenna. With two antennas, after performing Subband Permutation on the first subblock, we obtain 2 different OFDM sets of sequences. These are the original set

$\mathrm{X}_{1}=\left[X_{1}^{1}, X_{2}^{1}-------, X_{M}^{1}\right]$

and

$\mathrm{X}_{2}=\left[X_{1}^{2}, X_{2}^{2}-------, X_{M}^{2}\right]$

and the original set with the first subblock swapped;

$\mathrm{X}_{1}=\left[X_{0}^{2}, X_{1}^{1}-------, X_{M}^{1}\right]$

and

$\mathrm{X}_{1}=\left[X_{0}^{1}, X_{1}^{2}------, X_{M}^{2}\right]$

Same operations are then performed on all other subblocks. With $\Phi \mathrm{M} \Phi$ subblocks and 2 antennas, totally $M{ }^{2} \Phi$ permutated sequence sets can be obtained. Out of these $\mathrm{M}^{2}$ sequence sets, based on a certain criterion, a set $\leftarrow\left\{X_{1}, X_{2}\right\} \leftarrow$ with the best PAPR property is chosen for transmission. Here, we consider a minimum average (minaverage) criterion. For each of $\mathrm{M}^{2}$ sequence sets, we first calculate the PAPR of $X_{1}$ and $X_{2}$ and denote it as

$\operatorname{PAPR}\left(X_{1}\right), \quad \operatorname{PAPR}\left(X_{2}\right)$

denote it as mean $\left\{\operatorname{PAPR}\left(X_{1}\right), \operatorname{PAPR}\left(X_{2}\right)\right\}$.

After identifying averages for all $\mathrm{M}^{2}$ sequence sets, we pick a set with the minimum mean $\left\{\operatorname{PAPR}\left(X_{1}\right), \operatorname{PAPR}\left(X_{2}\right)\right\}$ for transmission.

\section{SIMULATION RESULT}

To calculate the PAPR performance accurately from the statistical point of view, the complementary cumulative distribution function (CCDF) of the PAPR of the OFDM signals is used to describe the probability of exceeding a given threshold $\mathrm{PAPR}_{0}, \mathrm{CCDF}=\operatorname{Pr}(\mathrm{PAPR}>\mathrm{PAPR})$.

In a simple approximate expression is derived for the CCDF of the PAPR of a multicarrier signal $\operatorname{Pr}($ PAPR $>$ PAPR $)=1-\left(1-\exp \left(-\mathrm{PAPR}_{0}\right)\right)^{\mathrm{N}}$.

In the results which follow, $10^{5}$ random OFDM sequences were generated to obtain the CCDF's. We use two transmit antennas and $\mathrm{N}=64$ subcarriers. The input constellation used is 4-QAM.

Figure 3, Figure 4 and Figure 5 shows, the PAPR vs CCDF of the the proposed SLM method for the case of two transmit antennas, for $\mathrm{u}=4$, and $\mathrm{u}=6$ at different values of $\mathrm{M}=2,4,6$ and 8 respectively. There are several observations that can be made from These plots. In the table there is comparison of PAPR vs CCDF of the the SLM methods for the case of two transmit antennas, for $\mathrm{u}=4$ and $\mathrm{u}=6$

\begin{tabular}{|l|l|l|}
\hline Original SLM Technique & $\begin{array}{l}\text { SLM scheme with subband } \\
\text { permutation }\end{array}$ & Proposed SLM technique \\
\hline $8.1 \mathrm{~dB}$, for $\mathrm{u}=4$ & $\begin{array}{l}6.3,6.15 \text { and } 6.1, \mathrm{~dB}, \text { for } \\
\mathrm{M}=2,4,8 \text { at fixed } \mathrm{u}=4\end{array}$ & $\begin{array}{l}\text { 5, 3.3 and } 2 \text { 1.6 } \mathrm{dB} \text { for } \mathrm{M}=2,4,6,8 \\
\text { at fixed } \mathrm{u}=4\end{array}$ \\
\hline $7.6 \mathrm{~dB}$, for $\mathrm{u}=6$ & $\begin{array}{l}6.1,5.8 \text { and } 5.7 \mathrm{~dB} \text { form }=2,4,8 \\
\text { at fixed } \mathrm{u}=6\end{array}$ & $\begin{array}{l}4.2,2.5 \text { and } 1.3,1.0 \mathrm{~dB} \text { for } \\
\mathrm{M}=2,4,6,8 \text { at fixed } \mathrm{u}=6\end{array}$ \\
\hline
\end{tabular}

Firstly, the proposed scheme achieves significantly better performance than the original SLM scheme and SLM scheme with subband permutation.
From figure 3 , where $\mathrm{u}$ is fixed at 2 the PAPR reduction gain of the proposed method is about $3.5 \mathrm{~dB}$ for $M=2$ and $6.2 \mathrm{~dB}$ for $M=4$ and $7.4 \mathrm{~dB}$ for $M=6$ compared with the SLM Scheme. This SLM based 
International Journal of Trend in Scientific Research and Development (IJTSRD) ISSN: 2456-6470 PAPR reduction gives better performance as compared to others PAPR reduction techniques.

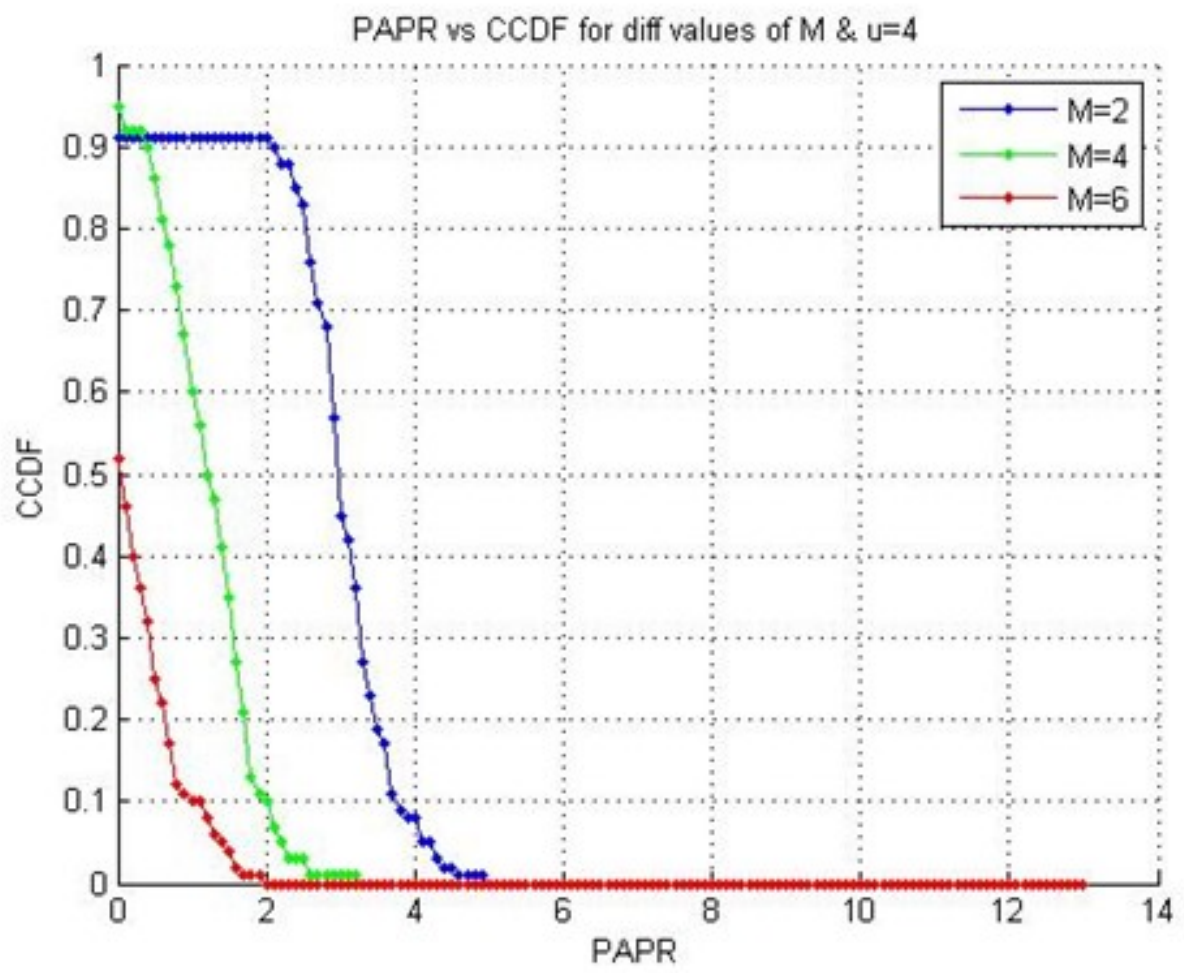

Figure 3 (PAPR vs CCDF for different value of $M \& u=4)$

From figure 4, where $\mathrm{u}$ is fixed at 4 the PAPR reduction gain of the proposed method is about $3.1 \mathrm{~dB}$ for $M=2$, $4.8 \mathrm{~dB}$ for $M=4$ and $6.1 \mathrm{~dB}$ for $M=6$ and $\mathrm{M}=8$ compared with the SLM techniques.

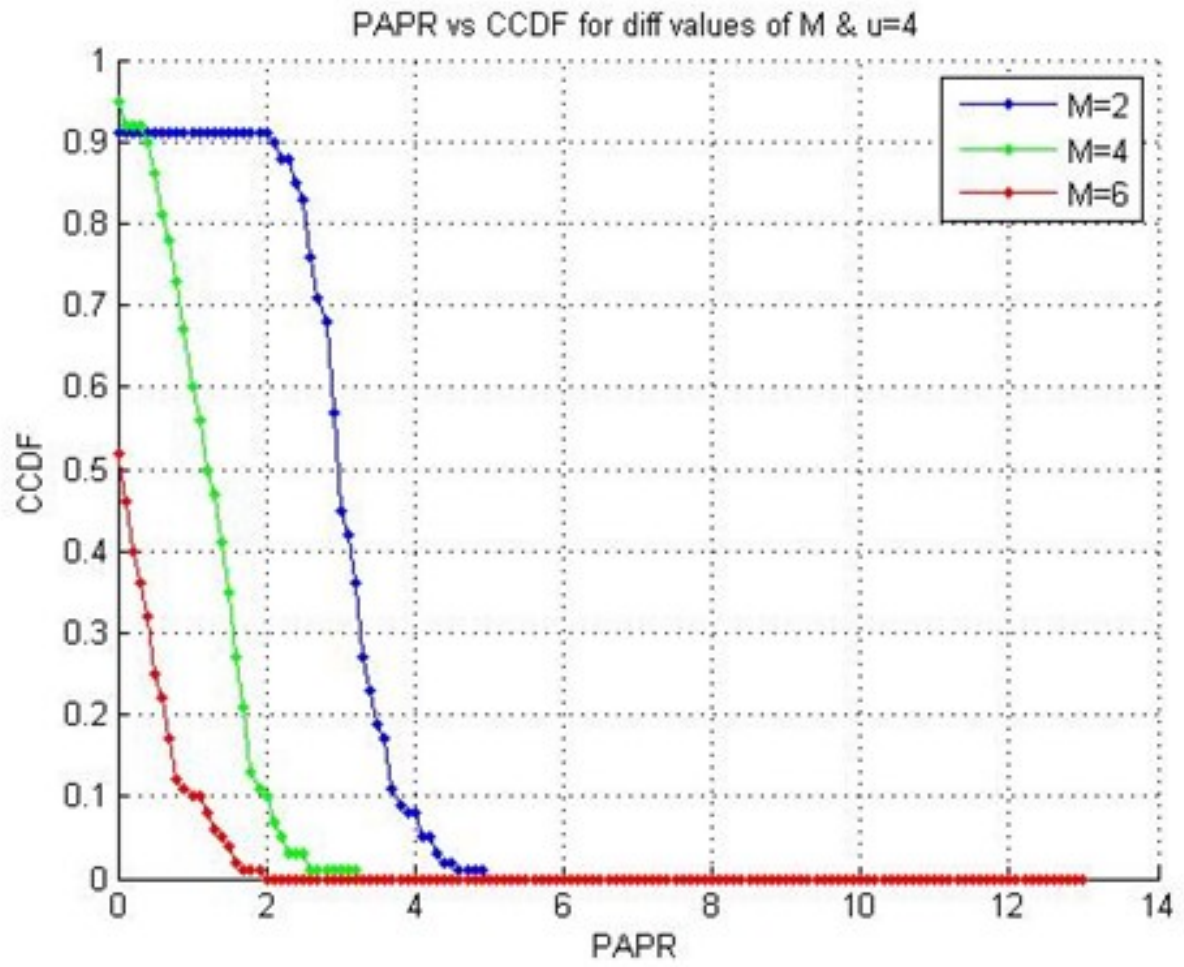

Figure 4 (PAPR vs CCDF for different value of $M \& u=4$ ) 
From figure 5, where $\mathrm{U}$ is fixed at 6 the PAPR reduction gain of the proposed method is about $3.1 \mathrm{~dB}$ for $M=$ 2, $4.8 \mathrm{~dB}$ for $M=4$ and $6.1 \mathrm{~dB}$ for $M=6$ and $\mathrm{M}=8$ compared with the SLM

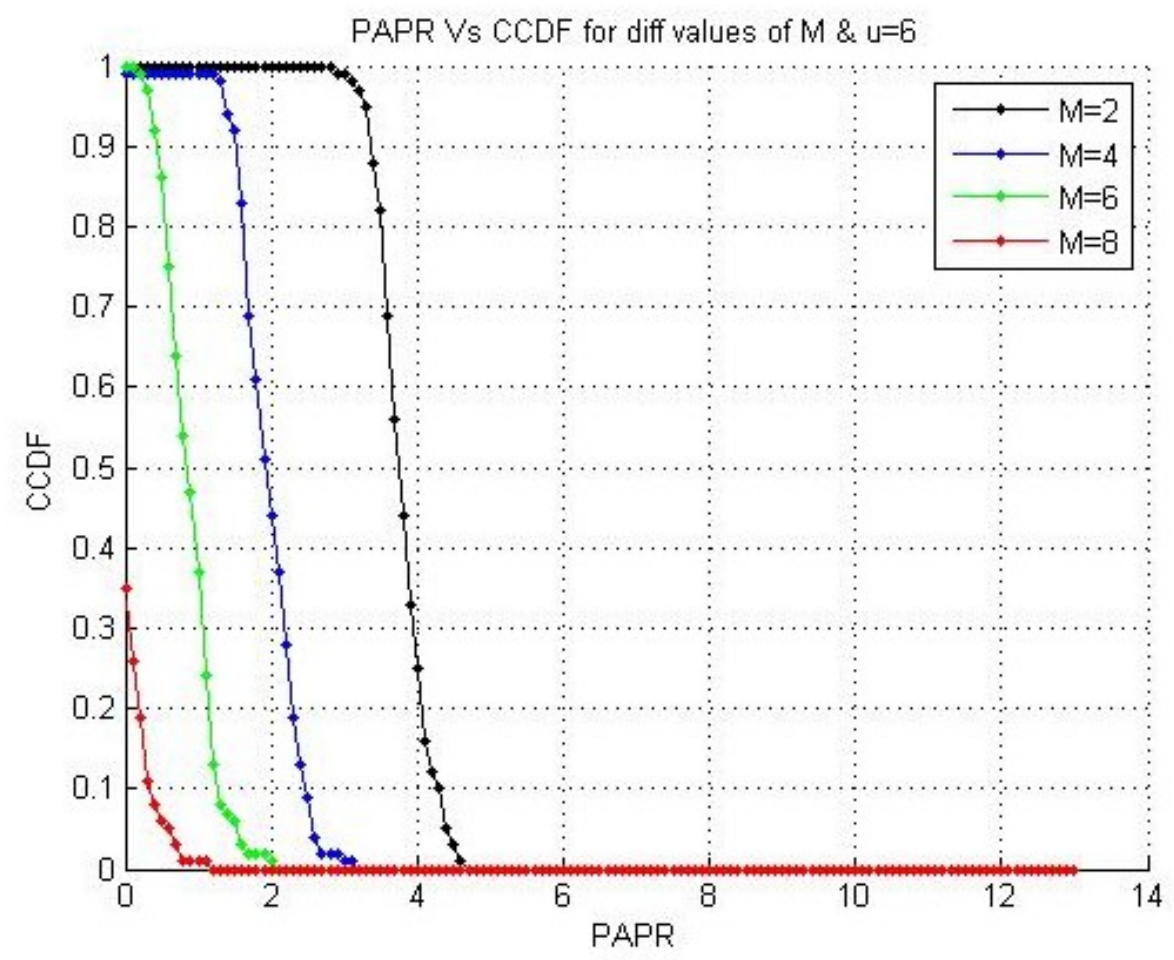

\section{Figure 5 (PAPR vs CCDF for different value of $M \& u=6$ )}

\section{CONCLUSION}

The disadvantages of the OFDM systems that is a high peak to average power ratio, namely the peak value of some of the transmitted signals could be much larger than the typical values. PAPR makes the amplifiers to work in non-linear regions This will cause inter modulation between the different sub carriers and introduce additional interference to the system. Additional interference leads to an increase in Bit Error Rate (BER).Large PAPR leads to inband distortion and spectral spreading There are number of techniques to deal with the problem of PAPR Some of them are amplitude clipping, filtering, coding, partial transmit sequence and selected mapping (SLM) and partial transmit sequences(PTS). In this paper a new selected mapping method (SLM) is given that provides good performance for PAPR reduction a more efficient solution using SLM in combination with sub band Permutation is proposed for STBC MIMO-OFDM Systems. The proposed method was shown to have a 5-7 dB PAPR reduction gain for 2 transmit antennas, compared with the SLM scheme. To have a better compromise between the complexity of the system and gain in comparison with the SLM method, the following value of $M=4,6$ and 8 can be used for For $\mathrm{u}=4$ and $\mathrm{u}=6, \mathrm{M}=2$ can be used. Our simulations have demonstrated that, considerable performance improvement can be achieved by the proposed PAPR-reduction method and PAPR reduction using this new SLM technique is better results as compared with the conventional SLM scheme

\section{REFERENCES}

1. S. HEE HAN, AND J. HONG LEE, "Modified Selected Mapping Technique for PAPR Reduction of Coded OFDM Signal" IEEE transactions on broadcasting, vol. 50, no. 3, september [2004].

2. S. HEE HAN, J. HONG LEE, “an overview of peak-to-average power ratio reduction techniques for multicarrier transmission" IEEE wireless

Communications April [2005]

3. R.O'Neill and L. B. Lopes, "Envelope Variations and Spectral Splatter in Clipped Multicarrier Signals," Proc. IEEE PIMRC '95,Toronto, Canada, Sept. [1995]

4. J. Armstrong, "Peak-to-average power reduction for OFDM by repeated clipping and frequency domain filtering," IEEE vol. 38, no. 5, pp. 246247, Feb. [2002]. 
5. K. G. Paterson and V. Tarokh, "On the Existence and Construction of Good Codes with Low Peakto-Average Power Ratios," IEEE Trans.Info. Theory, vol. 46, no. 6, Sept. [2000]

6. B. S. Krongold and D. L. Jones, "An Active-Set Approach for OFDM PAPR Reduction via Tone Reservation," IEEE Trans. Signal Processing, vol. 52, no. 2, pp. 495-509, Feb. 2004.

7. C. Tellambura, "Computation of the ContinuousTime PAR of an OFDM Signal with BPSK Subcarriers," IEEE Commun. Lett., vol . 5, no. 5, May 2001, pp. 185-87.

8. M. Tan, Z. Latinovi'c, and Y. Bar-Ness, "STBC MIMO-OFDM Peakto-Average Power Ratio Reduction by Cross-Antenna Rotation and Inversion," IEEE COMMUNICATIONS LETTERS, VOL. 9, NO. 7,JULY 2005.

9. R. van Nee and R. Prasad, OFDM for Wireless Multimedia Communications, Artech House, 2000 .

10. S. Hee Han, and J. Hong Lee, "Modified Selected Mapping Technique for PAPR \Reduction of Coded OFDM - Signal" - IEEE TRANSACTIONSON BROADCASTING, VOL. 50, NO. 3, SEPTEMBER 2004.

11. S. HEE HAN, J. HONG LEE, "an overview of peak-to-average power ratio reduction techniques for multicarrier transmission" IEEE WIRELESS Communications April 2005

12. R. O'Neill and L. B. Lopes, "Envelope Variations and Spectral Splatter in Clipped Multicarrier Signals," Proc. IEEE PIMRC 195, Toronto, Canada, Sept. 1995, pp. 71-75.

13. J. Armstrong, "Peak-to-average power reduction for OFDM by repeated clipping and frequency domain filtering," IEE Electr. Lett., vol. 38, no. 5, pp. 246-247, Feb. 2002.

14. K. G. Paterson and V. Tarokh, "On the Existence and Construction of Good Codes with Low Peakto-Average Power Ratios," IEEE Trans. Info. Theory, vol. 46, no. 6, Sept. 2000, pp. 1974-87.

15. B. S. Krongold and D. L. Jones, "An Active-Set Approach for OFDM PAR Reduction via Tone Reservation," IEEE TRANS. SIGNAL PROCESSING, VOL. 52, NO. 2, PP. 495-509, FEB. 2004.
16. B. S. Krongold and D. L. Jones, "PAR Reduction in OFDM via Active Constellation Extension," IEEE Trans. Broadcast., vol. 49, no. 3, Sept. 2003, pp. 258-68.

17. A. D. S. Jayalath and C. R. N. Athaudage, "On the PAR Reduction of OFDM Signals Using Multiple Signal Representation," IEEE Commun. Lett., vol. 8, no. 7, pp. 425-427, July 2004.

18. S. H. Han and J. H. Lee, "PAPR Reduction of OFDM Signals Using a Reduced Complexity PTS Technique," IEEE Sig. Proc. Lett., vol. 11, no. 11, Nov. 2004, pp. 887-90.

19. Chin-Liang Wang and Yuan Ouyang,"LowComplexity Selected Mapping Schemes for Peakto-Average Power Ratio Reduction in OFDM Systems, "IEEE transactions on signal processing, vol. 53, no. 12, december 2005

20. D.S. Jayalath and C. Tellambura, "The use of interleaving to reduce the peak-to-average power ratio of an OFDM signal," IEEE GLOBECOM'00, pp.82-86, 2000

21. B. S. Krongold and D. L. Jones, "PAR Reduction in OFDM via Active Constellation Extension," IEEE Trans. Broadcast., vol. 49, no.3, Sept. [2003].

22. A. D. S. Jayalath and C. R. N. Athaudage, "On the PAR Reduction of OFDM Signals Using Multiple Signal Representation," IEEE Commun. Lett., vol. 8, no. 7, pp. 425-427, July [2004].

23. S. H. Han and J. H. Lee, "PAPR Reduction of OFDM Signals Using a Reduced Complexity PTS Technique," IEEE Sig. Proc. Lett., vol. 11,no. 11, Nov. [2004].

24. Chin-Liang Wang and Yuan Ouyang,"LowComplexity Selected Mapping Schemes for Peakto-Average Power Ratio Reduction in OFDM Systems," IEEE Transactions on signal processing, vol. 53, no. 12, December [2005]

25. A.D.S. Jayalath and C. Tellambura, "The use of interleaving to reduce the peak-to-average power ratio of an OFDM signal," IEEE GLOBECOM'00, pp.82-86, [2000].

26. Ben Jmâa Ahmed Bassem, Jarboui Slaheddine and Bouallegue Ammar Laboratory Sys Com Ecole Nationale D'Ingénieurs de Tunis ENIT Tunis, Tunisia, 